\title{
Correction to: The test of both worlds: identifying feature binding and control processes in congruency sequence tasks by means of action dynamics
}

\author{
Stefan Scherbaum ${ }^{1}\left[\right.$ Dimon Frisch ${ }^{1} \cdot$ Maja Dshemuchadse ${ }^{1} \cdot$ Matthias Rudolf $^{1} \cdot$ Rico Fischer $^{1,2}$
}

Published online: 13 March 2020

๑) Springer-Verlag GmbH Germany, part of Springer Nature 2020

\section{Correction to: Psychological Research (2018) 82:337-352 https://doi.org/10.1007/s00426-016-0823-9}

An error in the description of one regressor and in the scaling of beta-weights lead to an incorrect figure and incorrect summary values in a table. Statistical analysis and interpretation referred to timing and not to absolute scale and is hence unaffected by the following corrections. The description of the regressor conflict adaptation on page 346 should read "With respect to the beta-weights resulting from the regressor conflict adaptation, a positive beta weight indicates a support of the response selection process by conflict adaptation in the current trial (leading to a more direct movement to the correct response box) while a negative beta weight indicates an impediment of the response selection process by conflict adaptation." (words in italics adapted) to fit to Table 1 "Coding of regressors" and to the general statement "all regressors were coded with ... higher values indicating more support for the correct Response"(p 345f).

Accordingly the beta-weight curve of conflict adaptation in Figure 7 ( $p$ 348) needs to be reversed. Moreover all betaweights in this figure must be scaled down by a factor of 2 , resulting in the following figure.

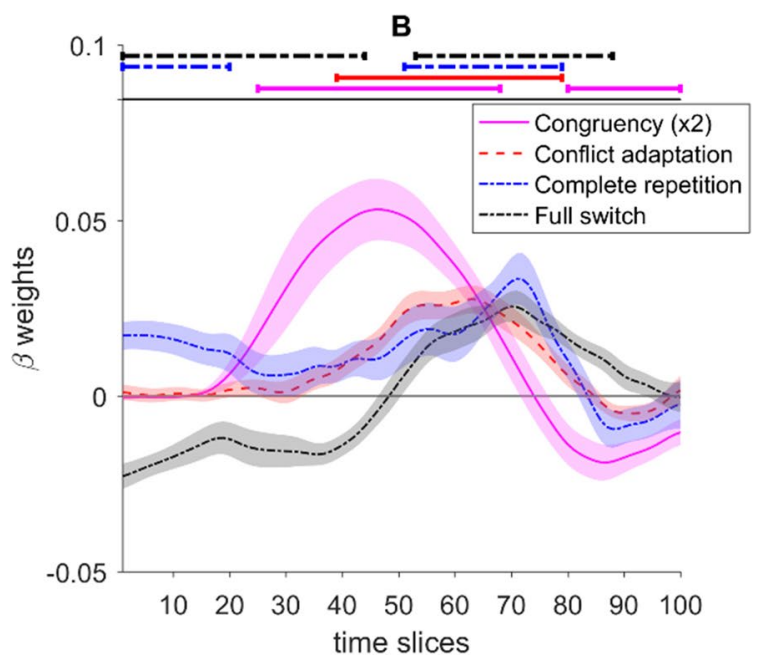

The area under the curve values for beta-weights in Table 2 (page 348) also need to be scaled down by factor 2 resulting in the following Table (adapted values in italics).

Department of Psychology, Ernst Moritz Arndt University of Greifswald, Greifswald, Germany

Stefan Scherbaum

Stefan.Scherbaum@tu-dresden.de

1 Department of Psychology, Technische Universita“t Dresden, Zellescher Weg 17, 01062 Dresden, Germany 
Table 2 Timing profiles of factors of influence on mouse movements yielded by time continuous multiple regression analysis with a full model including all four regressors in one step (left part) and a step-wise model with paired regressors entered successively in two steps (right part)

\begin{tabular}{|c|c|c|c|c|c|c|}
\hline & \multicolumn{3}{|c|}{ Full regression } & \multicolumn{3}{|c|}{ Step wise regression } \\
\hline & Time slice & Time & $\mathrm{AUC}($ mean $\pm \mathrm{SE})$ & Time slice & Time & $\mathrm{AUC}($ mean $\pm \mathrm{SE})$ \\
\hline \multirow[t]{2}{*}{ Congruency } & {$[25,69]$} & {$[145 \mathrm{~ms}, 400 \mathrm{~ms}]$} & $1.763 \pm 0.26$ & {$[25,68]$} & [145 ms, $394 \mathrm{~ms}]$ & $1.749 \pm 0.26$ \\
\hline & {$[80,100]$} & [464 ms, $580 \mathrm{~ms}]$ & $-0.329 \pm 0.09$ & {$[80,100]$} & [464 ms, $580 \mathrm{~ms}]$ & $-0.329 \pm 0.09$ \\
\hline $\begin{array}{l}\text { Conflict adapta- } \\
\text { tion }\end{array}$ & {$[40,78]$} & [232 ms, $452 \mathrm{~ms}]$ & $0.754 \pm 0.08$ & {$[39,79]$} & [226 ms, $458 \mathrm{~ms}]$ & $0.808 \pm 0.09$ \\
\hline \multirow{2}{*}{$\begin{array}{l}\text { Complete repeti- } \\
\text { tion }\end{array}$} & {$[1,19]$} & {$[6 \mathrm{~ms}, 110 \mathrm{~ms}]$} & $0.311 \pm 0.09$ & {$[1,20]$} & {$[6 \mathrm{~ms}, 116 \mathrm{~ms}]$} & $0.308 \pm 0.08$ \\
\hline & {$[66,78]$} & [383 ms, $452 \mathrm{~ms}]$ & $0.249 \pm 0.07$ & {$[51,79]$} & [296 ms, $458 \mathrm{~ms}]$ & $0.654 \pm 0.14$ \\
\hline \multirow[t]{2}{*}{ Full switch } & {$[1,44]$} & [6 ms, $255 \mathrm{~ms}]$ & $-0.662 \pm 0.14$ & {$[1,44]$} & [6 ms, $255 \mathrm{~ms}]$ & $-0.688 \pm 0.13$ \\
\hline & {$[53,88]$} & [307 ms, $510 \mathrm{~ms}]$ & $0.634 \pm 0.09$ & {$[53,88]$} & {$[307 \mathrm{~ms}, 510 \mathrm{~ms}]$} & $0.649 \pm 0.09$ \\
\hline
\end{tabular}

Entries in the table show segments (with [start, end] points) matching the significance criterion of the continuous regression analyses (i.e., segments with more than ten consecutive significant $t$ tests against zero). Segment times represent the projection of time slices to each participant's mean RT. AUC refers to the summed area under the curve for the respective segments and indicates the strength of the respective influence

Publisher's Note Springer Nature remains neutral with regard to jurisdictional claims in published maps and institutional affiliations. 